\title{
EVALUATION OF SALIVARY VERSUS SERUM SQUAMOUS CELL CARCINOMA ANTIGEN IN ORAL LICHEN PLANUS
}

\author{
Maha Abdelkawy*, Ahmed Nabil Fahmi**, Olfat shaker ${ }^{* * * *}$ and Shereen Ali ${ }^{* * * *}$
}

\begin{abstract}
Background and Objectives: Oral Lichen Planus is a chronic, inflammatory, immune mediated disease affecting the oral cavity. It is the oral variant of the mucocutaneous disease lichen Planus. The pathogenesis of OLP has been deeply studied yet not totally clear, this retrospective case control study aimed to evaluate squamous cell carcinoma antigen in serum and saliva of Oral lichen planus.

Methods: 30 individuals were included in the study; divided equally to two groups; Oral lichen planus and healthy volunteers. Serum and salivary samples were collected from patients and volunteers. Pain score was performed using Visual Analogue Scale. Thongprasom sign scoring for OLP was used to record the cases clinically; Oral lichen planus diagnosis was confirmed by histopathology. A double-antibody sandwich enzyme-linked immunosorbent assay was used to assay the level of Human squamous cell carcinoma antigen in samples.
\end{abstract}

Results: Both SccAg in serum and saliva showed statistically significantly higher mean levels than control group. Receiver Operating Characteristic curve analysis of the two markers showed $100 \%$ diagnostic accuracy on differentiation from control group.

Conclusion: Salivary SccAg can be considered as a significant diagnostic test for OLP disease activity.

\section{INTRODUCTION}

Oral Lichen Planus (OLP) is a chronic, inflammatory, immune mediated disease affecting the oral cavity. It is the oral variant of the mucocutaneous disease lichen Planus. OLP affects $1-2 \%$ of the adult population, with female predilection almost twice the male. It has six different clinical forms, reticular, plaque, papular, bullous, erosive/ulcerative, and atrophic. These are either seen together or independently. The bilateral presentation and presence of Wickham's stria are the classical presentation of the disease. It's characterized by remission and exacer-

\footnotetext{
* Department of Oral Medicine, and Periodontology, Faculty of Dentistry, Beni-Suef University, Beni-Suef, Egypt.

** Department of Oral Pathology, Faculty of Dentistry, Beni-Suef University, Beni-Suef, Egypt.

*** Department of Biochemistry, Faculty of Medicine, Cairo University.

**** Department of Oral Medicine and Periodontology, Faculty of Oral and Dental Medicine, Cairo University.
} 
bation; during exacerbation state the symptoms and signs highly increase while in remission the opposite occurs. ${ }^{(1,2)}$

The histopathological criteria of the disease involve; basal cell liquefaction degeneration, colloid bodies, dense band-like infiltration of lymphocytes along the interface between epithelium and connective tissue, sawtooth like rete pegs and hyperkeratosis. ${ }^{(1)}$.

The pathogenesis of OLP has been deeply studied yet not totally clear, It's mechanism has been described to be either specific where T-cells play a major role, and nonspecific where cytokines, matrix metalloproteinases and other immune cells mediate it. ${ }^{(3)}$

Squamous cell carcinoma antigen ( $\mathrm{SccAg}$ ) is an inhibitor of serine protease inhibitor. It is found in normal squamous epithelium in the spinous and granular layers. It is detected in epithelial origin neoplasms. It was first biochemically isolated from cervical squamous cell carcinoma by Kato and Torigoe ${ }^{(4)}$. SccAg has two forms; SccAg1 and SccAg2; derived from highly similar nearby genes on chromosome number eighteen. SccAg is found in biological fluids as mucus of respiratory system and saliva of healthy individuals as a product of passive secretion in the course of epithelial cells desquamation. ${ }^{(5)}$.

A recent review stated that although the best diagnosis for OLP is performed by biopsies, recognition of new biomarkers which are noninvasive are considered a rapid step to diagnose the disease in its beginning. Antioxidant and peroxidation levels, salivary cortisol levels, increase levels of immunoglobulin A and Immunoglobulin G, C-reactive protein, Matrix metalloproteineases- 8 and others were all suggested as potential biomarkers for early OLP diagnosis $^{(6,7)}$

Salivary biomarkers are now used in diagnosis, prognosis and monitoring disease activities in several different diseases including oral cancer and other types of cancer ${ }^{(8)}$. Hence, the purpose of this study is to evaluate salivary SccAg versus serum SccAg to be used as a biomarker for OLP.

\section{SUBJECTS AND METHODS}

This retrospective case control study was approved by the Research Ethics Committee of Faculty of Dentistry Beni-Suef University (ID \#FDBSUREC/27012019/AM) and was conducted in full accordance with the World Medical Association Declaration of Helsinki 1975, revised in 2003. Fifteen OLP patients and fifteen healthy volunteers comprising the control group participated in the study. The patients were randomly selected from the diagnostic center, Faculty of Dentistry, Cairo University in Cairo, Egypt. Each patient received the appropriate treatment after finishing their role in the study.

Exclusion Criteria: The criteria to exclude subjects from the study included: (1) pregnant or lactating females; (2) subjects having systemic disorder or taking medications; (3) subjects who suffer from any other mucosal lesions; and (4) severe periodontal inflammation.

The age of the study subjects was in the range of 24-60 years. They were divided into two groups; Control group with fifteen healthy volunteers; OLP group with fifteen symptomatic OLP patients. The diagnosis was confirmed by histopathology. OLP patients were not under any current medication and not suffering from any other systemic condition or any other oral mucosal disorder (based on clinical examination).

Pain score was performed using Visual Analogue Scale (VAS) where " 0 " represented absence of pain and "10" the worst pain. The patient was asked to score pain using a 0-to-10 Visual Analogue Scale (VAS) with "0" representing the absence of pain and " 10 " the worst pain ever ${ }^{(9)}$. Thongprasom sign scoring for OLP was used; 
Score 1:- mild white striae only.

Score 2:- White striae with atrophic area $<1 \mathrm{~cm}^{2}$.

Score 3:- White striae with atrophic area $>1 \mathrm{~cm}^{2}$.

Score 4: - White striae with erosive area $<1 \mathrm{~cm}^{2}$.

Score 5: - White striae with erosive area $>1 \mathrm{~cm}^{2}$, to record the cases clinically ${ }^{(10)}$

\section{Collection of salivary samples}

Whole unstimulated saliva had been collected from all participants by means of standard techniques defined by Navazesh (11). Prior to assessment, Subjects were requested to stop eating $\&$ drinking for minimum half an hour. Collection of samples was carried out by asking individuals to first swallow then tilt their head forward to expectorate saliva in a tube. This was done for 5 minutes. Obtained samples were then stored at $-20^{\circ} \mathrm{C}$ till assessed.

\section{Blood sample collection:}

Three milliliter blood was collected from each subject in ethylenediaminetetraacetic acid (EDTA) tubes by venipuncture at the antecubital fossa. The tubes were maintained in ice and transported to the biochemistry lab at Faculty of Medicine, Cairo University, where it was stored at $-70^{\circ} \mathrm{C}$ until all samples were collected. All collected samples were anonymous given specific serial number for blinding.

\section{Determination of squamous cell carcinoma related antigen in serum and saliva:}

Serum and saliva were used for determination of SccAg by enzyme-linked immunosorbent assay (ELISA). The ELISA kit was provided by Bioassay Technology Laboratory; Shanghai, China (Cat.No$\mathrm{E} 2108 \mathrm{Hu})$.

The kit uses a double-antibody sandwich to assay the level of Human SccAg in samples. Add SccAg to monoclonal antibody Enzyme well which is pre-coated with $\mathrm{SccAg}$ monoclonal antibody, incubation; then, add SccAg antibodies labeled with biotin, and combined with Streptavidin-HRP to form immune complex; then carry out incubation and washing again to remove the uncombined enzyme. Then add Chromogen Solution A, B, the color of the liquid changes into the blue, and at the effect of acid, the color finally becomes yellow. The chroma of color and the concentration of the Human Substance SccAg of sample were positively correlated to each well to stop the reaction (the blue color was changed into yellow immediately).

\section{Sample size calculation}

This power analysis used Area Under the Receiver Operating Characteristic (ROC) curve (AUC) of SccAg as the primary outcome. Since no relevant literature was found for SccAg in serum and saliva of patients with and Oral LP, the results of Watanabe et al (12) were used for sample size calculation. Area under ROC curve was 0.9 based upon paper results against the null hypothesis value of 0.5. Using alpha $(\alpha)$ level of $(5 \%)$ and Beta $(\beta)$ level of $(20 \%)$ i.e. power $=80 \%$; the minimum estimated sample size was 15 subjects per group. Sample size calculation was performed using MedCalc Version 11.3.0.0.

\section{Statistical Analysis}

Numerical data were explored for normality by checking the distribution of data and using tests of normality (Kolmogorov-Smirnov and ShapiroWilk tests). All data showed normal (parametric) distribution except for OLP pain and clinical signs scores which showed non-normal (non-parametric) distribution. Data were presented as mean, standard deviation (SD) and 95\% Confidence Interval (95\% $\mathrm{CI})$ values. One-way ANOVA was used to compare between the three groups. Bonferroni's post-hoc test was used for pair-wise comparisons when ANOVA test is significant. Pearson's and Spearman's correlation coefficients were used to determine significant correlations between different variables. 
Qualitative data were presented as frequencies and percentages. Fisher's Exact test was used for the comparisons. ROC curves were constructed to determine the cut-off values of different markers for differentiation between the different groups. Areas under the ROC curve (AUCs) were compared using $z$-statistic. The significance level was set at $P \leq 0.05$. Statistical analysis was performed with IBM SPSS Statistics for Windows, Version 23.0. Armonk, NY: IBM Corp.. ROC curve analysis was performed with MedCalc Version 11.3 for Windows (MedCalc Software bvba).

\section{RESULTS}

\section{Base line characteristics}

There was no statistically significant difference between gender or age distributions in the two groups. Table (1)

TABLE (1): Descriptive statistics and results of comparisons between base line characteristics in the two groups

\begin{tabular}{|c|c|c|c|c|}
\hline & & $\begin{array}{c}\text { LP } \\
(\mathrm{n}=15)\end{array}$ & $\begin{array}{c}\text { Control } \\
(\mathrm{n}=15)\end{array}$ & P-value \\
\hline \multirow{2}{*}{$\begin{array}{c}\text { Age } \\
\text { (Years) }\end{array}$} & $\begin{array}{c}\text { Mean } \\
(\mathrm{SD})\end{array}$ & $42.3(11)^{\text {В }}$ & $38.5(7.8)^{\text {В }}$ & \multirow{2}{*}{$0.001^{*}$} \\
\cline { 2 - 4 } & $95 \% \mathrm{CI}$ & $36.2-48.4$ & $34.2-42.9$ & \\
\hline \multirow{2}{*}{$\begin{array}{c}\text { Gender } \\
\text { [n (\%)] }\end{array}$} & Male & $\begin{array}{c}3 / 15 \\
(20 \%)\end{array}$ & $\begin{array}{c}4 / 15 \\
(26.7 \%)\end{array}$ & \multirow{2}{*}{0.365} \\
\cline { 2 - 4 } & Female & $\begin{array}{c}12 / 15 \\
(80 \%)\end{array}$ & $\begin{array}{c}11 / 15 \\
(73.3 \%)\end{array}$ & \\
\hline
\end{tabular}

*: Significant at $P \leq 0.05$, Different superscripts in the same row are statistically significantly different

\section{Comparison between groups}

As regards for both SccAg in serum and saliva; OLP group showed statistically significantly higher mean SccAg levels than control group in both serum and saliva. Table (2)
TABLE (2): Descriptive statistics and results of one-way ANOVA test for comparisons between SccAg levels in the three groups

\begin{tabular}{|c|c|c|c|}
\hline & $\begin{array}{c}\text { LP } \\
(n=15)\end{array}$ & $\begin{array}{l}\text { Control } \\
(\mathrm{n}=15)\end{array}$ & P-value \\
\hline \multicolumn{4}{|c|}{ SccAg in serum $(\mathrm{ng} / \mathrm{ml})$} \\
\hline Mean (SD) & $4(1.39)^{\mathrm{A}}$ & $0.92(0.26)^{\mathrm{B}}$ & \multirow{2}{*}{$<0.001 *$} \\
\hline $95 \% \mathrm{CI}$ & $3.23-4.77$ & $0.77-1.06$ & \\
\hline \multicolumn{4}{|c|}{ SccAg in saliva $(\mathrm{ng} / \mathrm{ml})$} \\
\hline Mean (SD) & $6.19(0.68)^{\mathrm{A}}$ & $2.09(0.4)^{\mathrm{B}}$ & \multirow{2}{*}{$<0.001 *$} \\
\hline $95 \% \mathrm{CI}$ & $5.81-6.56$ & $1.87-2.32$ & \\
\hline
\end{tabular}

*: Significant at $P \leq 0.05$, Different superscripts in the same row are statistically significantly different

\section{Correlation analysis}

There was no statistically significant difference between different variables. Table (3)

TABLE (3): Results of Pearson's and Spearman's correlation coefficients for the correlation between different variables

\begin{tabular}{|c|c|c|}
\hline & $\begin{array}{c}\text { LP } \\
(n=15)\end{array}$ & $\begin{array}{l}\text { Control } \\
(\mathrm{n}=15) \\
\end{array}$ \\
\hline \multicolumn{3}{|l|}{ SccAg in serum and saliva } \\
\hline Pearson's Correlation coefficient & 0.160 & 0.092 \\
\hline P-value & 0.570 & 0.744 \\
\hline \multicolumn{3}{|l|}{ SccAg in serum and OLP pain score } \\
\hline Spearman's Correlation coefficient & -0.148 & \multirow{2}{*}{ - } \\
\hline $\mathrm{P}$-value & 0.599 & \\
\hline \multicolumn{3}{|c|}{ SccAg in serum and OLP clinical signs score } \\
\hline Spearman's Correlation coefficient & -0.210 & \multirow{2}{*}{ - } \\
\hline P-value & 0.452 & \\
\hline \multicolumn{3}{|l|}{ SccAg in saliva and OLP pain score } \\
\hline Spearman's Correlation coefficient & 0.181 & \multirow{2}{*}{ - } \\
\hline P-value & 0.518 & \\
\hline \multicolumn{3}{|c|}{ SccAg in saliva and OLP clinical signs score } \\
\hline Spearman's Correlation coefficient & -0.466 & \multirow{2}{*}{ - } \\
\hline $\mathrm{P}$-value & 0.080 & \\
\hline
\end{tabular}

*: Significant at $P \leq 0.05$ 


\section{Diagnostic accuracy of the two markers (ROC curve analysis)}

ROC curve analysis of the two markers for differentiation between LP and control groups showed a diagnostic accuracy $=100 \%$ at cut-off values of 2.6 and $1.5 \mathrm{ng} / \mathrm{ml}$ for $\mathrm{SccAg}$ in serum and saliva, respectively.

\section{DISCUSSION}

OLP is a common oral mucosal lesion with 0.5$2 \%$ prevalence in adult population. It classically presents as bilateral symmetrical lesion mostly on the buccal mucosa without excluding any other site. The atrophic and erosive/ulcerative lesions are the most clinical forms of OLP causing variable discomfort. ${ }^{(13)}$ Although OLP have been studied for several decades its etiology and pathogenesis has not been clearly understood yet. Many predisposing factors have been found to be associated with the diseases. ${ }^{(1)}$

SccAg is a subfraction of a tumor associated antigen in squamous cell carcinoma. It has two forms, the first, SccAg1, which is a neutral form protein that is expressed in all cells, while $\mathrm{SccAg} 2$ is an acidic form specifically secreted by malignant cells. The neutral SccAg subfraction remain in all cells $^{(14,15,16)}$.

This study demographic data showed no statistical significant difference for gender or age between the two groups. The results rev ealed highly significant levels of SccAg in OLP group compared to control group. This implies the high presentation of SccAg in OLP which might take a part in its pathogenesis that is not yet discovered. Proinflammatory cytokines were found to stimulate SccAg production. SccAg is thought to contribute in restoration of homeostasis through their action on proteolysis in inflamed epithelial cells ${ }^{(5)}$.

Our results were in consistent with Sun and Chiang who found that serum SccAg was significantly higher than control subjects in major types of erosive OLP. The levels of SccAg decreased on treatment of erosive OLP patients with levamisole plus Chinese medicinal herbs as well as each one alone. They stated that SccAg serum levels of treated erosive OLP dropped during remission phase and increased on exacerbation ${ }^{(17)}$. In our data, the correlation between pain and ulcers of OLP with serum and salivary SccAg were not found to be significant. This may be attributed to that most patients were symptomatic, on sample collection, and the sample size of the study.

On correlating serum and salivary SccAg in OLP, there was a weak positive relation. Salivary SccAg showed higher values but with no statistical significance. This states that increased serum SccAg is accompanied by increased salivary SccAg. This could be due to saliva being in direct contact with the OLP lesions that bare an inflammatory factor and all its outcomes, more accurately detecting the SccAg and hence representing altered protein level salivary biomarker. It also highlights that salivary SccAg could be reliable as serum and therefore more easily used and accepted.

The current research revealed that salivary and serum SccAg for OLP showed $100 \%$ diagnostic accuracy when differentiated from control group with 2.6 and $1.5 \mathrm{ng} / \mathrm{ml}$ as cut-off values for serum and saliva giving excellent sensitivity and specificity. To our knowledge no other study used the diagnostic accuracy as index test for SccAg as biomarker either salivary or serum for OLP using the ROC curve and plotting the sensitivity and specificity. A study performed on Psoriasis patients; which is an inflammatory chronic skin disease with increased proliferation of epidermis and infiltration of dermis with inflammatory cells; showed increased SccAg in serum of psoriasis patients that correlated with severity of the disease and reflected the treatment efficacy. They used the ROC curve analysis and the area under the curve between healthy controls and psoriasis patients was 0.877 with the cut-off value found at $1.05 \mathrm{ng} / \mathrm{ml}^{(12)}$. 
Recently, salivary biomarkers are being used for its potential diagnostic and screening features, especially for its noninvasive procedure, sensitivity, specificity and practicality as well as better patient compliance than blood biomarkers. It was formerly thought to have a major drawback due to its minute quantity components yet the emergence of new analytical techniques detecting small amount of proteins diminished this draw back ${ }^{(8)}$

In a case report $\mathrm{SccAg}$ was used as one of the standardization panel of blood biomarkers for monitoring oral squamous cell carcinoma, the authors adopted the idea of measuring more than one biomarker together and so enhancing the diagnostic accuracy of malignant analysis ${ }^{(18)}$. SccAg was found also to be a useful tumor marker as its presence in serum has been directly related to the clinical staging of oral squamous cell carcinoma. It was suggested that high level of SccAg might even have a prognostic value for oral squamous cell carcinoma ${ }^{(16)}$.

OLP is well known of being a chronic inflammatory disease, with small percentage of malignant transformation $(1.1 \%)^{(19)}$. Despite the small sample size of the current study, the results imply that high SccAg in saliva could aid in following the disease activity.

\section{CONCLUSION AND RECOMMENDATIONS}

- Salivary SccAg has shown to give same results as serum indicating that it could be reliable biomarker for disease activity.

- Studies with larger sample size targeting level of SccAg in different types of OLP are needed.

- Further studies with long follow up to detect if SccAg can be considered as a diagnostic test for OLP malignant transformation.

\section{Study limitations:}

1- Small sample size

2- Salivary biomarkers drawbacks as lack of standardization procedures and its validation in oral inflammatory lesions

\section{Practical implication:}

Salivary SccAg could be used as a diagnostic test for OLP disease activity

\section{Conflict of interest:}

All Authors declare no conflict of interest

\section{Funding:}

The study was funded by personal resources (self-funded)

\section{REFERENCES}

1. McCullough M, Alrashdan M, and Cirillo N.' Oral Lichen Planus'. Springer Nature Switzerland 2019. C Farah et al. Contemporary Oral Medicine. 1043-1077.

2. Cozzani E, Russo R, Mazzola F, Garofolo S, Camerino M, Burlando M, Peretti G, Parodi A. Narrow-band imaging: a useful tool for early recognition of oral lichen planus malignant transformation? Eur J Dermatol 2019; 29(5): 500-506.

3. Alrashdan M, Cirillo $\mathrm{N}$ and McCullough M. Oral lichen planus: a literature review and update. Arch Dermatol Res 2016 308:539-551.

4. Kato H, Torigoe T. Radioimmunoassay for tumor antigen of human cervical squamous cell carcinoma. Cancer 1977;40:1621-1628.

5. Hayat M., in Handbook of Immunohistochemistry and in Situ Hybridization of Human Carcinomas, London, Science direct,2005, vol. 3 .

6. Nosratzehi T. Oral Lichen Planus: an Overview of Potential Risk Factors,Biomarkers and Treatments. Asian Pacific Journal of Cancer Prevention, 2018, Vol 19 1161, 19 (5), 1161-1167.

7. Talungchit S, Buajeeb W, Lerdtripop C, Surarit R, Chairatvi K, Roytrakul S, et al. Putative salivary protein biomarkers for the diagnosis of oral lichen planus: a case control study. BMC Oral Health 2018 18-42.

8. Hegde ND, Hegde MN, Priya G An Insight on salivary biomarkers for oral Cancer. J Dent Craniofac Res. ,2018, Vol.3 No.2-8.

9. Gobbo M, Rupel K, Zoi V, Perinetti G, Ottaviani G, Di Lenarda R, Bevilacqua L, Woo SB, Biasotto M. Scoring systems for Oral Lichen Planus used by differently experienced raters. Med Oral Patol Oral Cir Bucal. 2017, Sep 1;22 (5):e562-571. 
10. Thongprasom K, Luangjarmekorn L, Sererat T, Taweesap W. Relative efficacy of fluocinolone acetonide compared with triamcinolone acetonide in treatment of oral lichen planus. J Oral Pathol Med. 1992;21:456-458.

11. Navazesh M. Methods for collecting saliva. Jam N.Y.Acad Sci. 1993. 20: 72-77.

12. Watanabe Y, Yamaguchi Y, Komitsu N, S. Ohta S, Azuma, Izuhara Yand Aihara M Elevation of serum squamous cell carcinoma antigen 2 in patients with psoriasis: associations with disease severity and response to the treatment. British Journal of Dermatology 2016, 174, pp1327-1336.

13. Gonzalez-Moles MA, Scully C, Gil-Montoya JA. Oral lichen planus: controversies surrounding malignant transformation. Oral Dis . 2008. 14:229-243.

14. Maruo T, Shibata K, Kimura A, Hoshina M, Mochizuki M. Tumor-associated antigen, TA-4, in the monitoring of the effects of therapy for squamous cell carcinoma of the uterine cervix. Serial determinations and tissue localization. Cancer 1985; 56:302-308.
15. Mino N, Iio A, Hamamoto K. Availability of tumor-antigen 4 as a marker of squamous cell carcinoma of the lung and other organs. Cancer 1988; 62: 730-4.

16. Lin W, Chen I, Wei F, et al. Clinical Significance of Preoperative Squamous Cell Carcinoma Antigen in Oral-Cavity Squamous Cell Carcinoma. Laryngoscope, 2011,121:971-977.

17. Sun A and Chiang C. Levamisole and/or Chinese medicinal herbs can modulate the serum level of squamous cell carcinoma associated antigen in patients with erosive oral lichen planus. J Oral Pathol Med 2001,30: 542-548.

18. Grimm M, Hoefert S, Krimmel M, Biegner T, Feyen O, Teriete $\mathrm{P}$ and Reinert S. Monitoring carcinogenesis in a case of oral squamous cell carcinoma using a panel of new metabolic blood biomarkers as liquid biopsies. Oral Maxillofac Surg ,2016, 20:295-302.

19. Aghbari S, Abushouk A, Attia A et al. Malignant transformation of oral lichen planus and oral lichenoid lesions: A meta-analysis of 20095 patient data. Oral Oncology 68.2017, 92-102. 